\title{
Chemotherapy Induced Lingual Hyperpigmentation
}

\author{
Iquebal Hasan ${ }^{1 *}$, BDS and Nicole Dolezar, MS $^{2}$ \\ ${ }^{1}$ Assistant Professor, Oral Medicine, Department of Surgical Sciences, East Carolina University School of Dental, Medicine, USA \\ ${ }^{2}$ Dental Student, East Carolina University School of Dental Medicine, USA
}

*Corresponding author: Iquebal Hasan, Assistant Professor, Oral Medicine, Department of Surgical Sciences, East Carolina University School of Dental, Medicine, USA.

Received Date: November 11, 2019

Published Date: December 16, 2019

\begin{abstract}
Patients receiving chemotherapy experience a variety of side effects in the oral cavity. Fortunately, most of them are short-term. One long-term side effect which has been reported due to chemotherapy is oral hyperpigmentation. This is challenging to diagnose due to a variety of conditions having similar clinical appearance. It can easily be misdiagnosed as racial pigmentation which is far more common. In this case report we will describe a 58-year-old African American female experiencing localized gum bleeding during brushing. She mentioned during the appointment that she also would like to get the hyperpigmentation on the dorsal surface of her tongue evaluated. The diagnosis of drug-induced pigmentary changes is based on correlating the onset of clinical appearance of pigmentation with initiation of chemotherapeutic drug use. The mechanism of chemotherapy-related oral hyperpigmentation remains unknown. However, lesion size measurements and photographs can be documented and tracked over time to rule out the possibility of melanoma. Patients should be made aware that oral hyperpigmentation due to chemotherapy is a benign condition.
\end{abstract}

Keywords: Hyperpigmentation; Chemotherapy; Oral; Tongue; Cyclophosphamide

\section{Introduction}

Many side effects are associated with chemotherapy treatment. Oral side effects may include mucositis, xerostomia, taste disturbances, candidiasis, and mucosal pigmentation [1] (Figure 1). Oral hyperpigmentation can be difficult to diagnose due to a variety of conditions having similar clinical appearance. It is important to consider the very common diagnosis of racial pigmentation. Pigmentation can be caused by a variety of drugs like, hormones, oral contraceptives, chemotherapeutic agents, anti-anxiety, antimalarial, anti-microbial and anti-fungal agents [2].

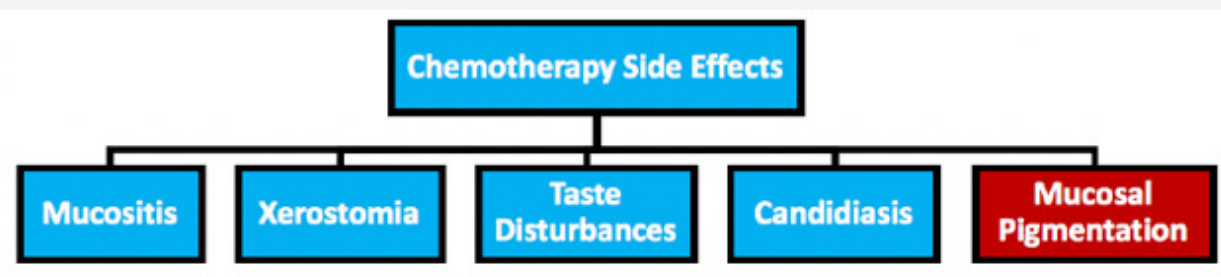

Figure 1: Chemotherapy treatment effects.

Chemotherapy drugs can cause hyperpigmentation, altering the pigmentation of skin, mucous membranes, and nails in generalized or localized areas [3,4]. Palate and gingiva are the most common sites affected. However, it can also be seen on the tongue. Darkened blotches on the tongue may appear as soon as 1or2 chemotherapy doses into treatment. Hyperpigmentation acquired in conjunction with chemotherapy may begin to fade between weeks to months after completion of treatment [4]. However, this can also remain long-term and become a permanent side-effect of chemotherapy.

\section{Case Report}

This article presents a 58-year-old African American female experiencing localized gum bleeding during brushing as well as 
hyperpigmentation on the tongue (Figure 2). Upon presentation, the patient's current medical status included hypercholesterolemia, diabetes, irritable bowel syndrome, and dissociative identity disorder. Her medication regimen included simvastatin and metformin. Past medical history included breast cancer with surgical history of C-section and modified radical mastectomy. Cyclophosphamide was the chemotherapeutic drug used to treat her breast cancer.

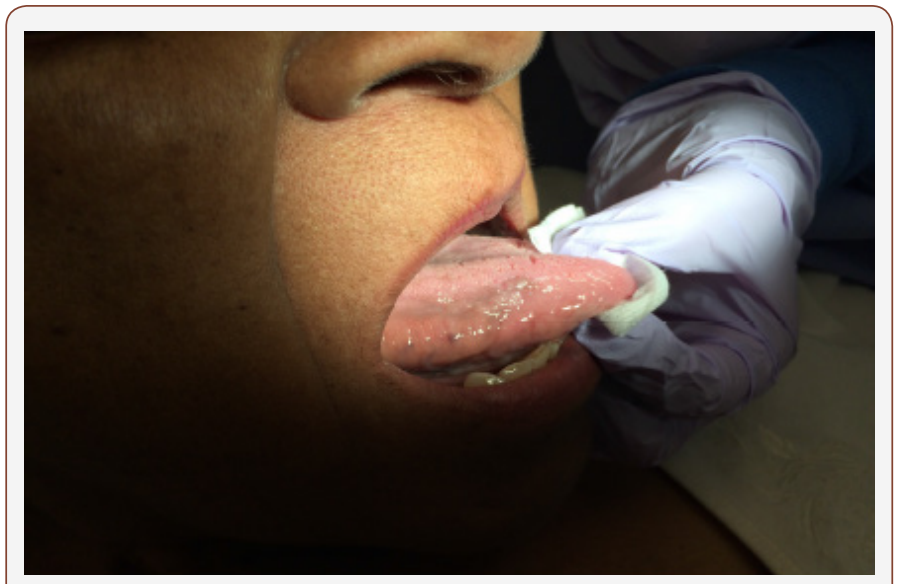

Figure 2: Tongue hyperpigmentation caused by chemotherapy with cyclophosphamide.

\section{Results}

Patient's lingual hyperpigmentation was previously diagnosed by her former dentist as racial pigmentation. Racial pigmentation of the oral cavity is usually observed in dark-skinned populations. This type of pigmentation ranges in color and location within the oral cavity. Color changes range from light to dark brown and is most commonly located within the gingiva and buccal mucosa. Other reported sites include lips, palate, and tongue [5]. It is crucial to correlate pigmentary changes with time of presentation onset to rule out the possibility of racial pigmentation. Environmental, physiological, and pathological causes should all be considered during development of a differential diagnosis for oral hyperpigmentation. It is important to note the patient's medical history in conjunction with the symptoms presented to look for a possible correlation. Diagnosis of drug-induced pigmentary changes is this case was based on correlating the onset of presentation with the initiation of chemotherapeutic drug use.

\section{Discussion}

There are certain side-effects associated with chemotherapy that effect the oral cavity in a variety of ways. These side effects can be classified into two categories: short term and long term. Fortunately, the majority of the side effects are short term which do not last more than a few weeks after completion of chemotherapy. Hyperpigmentation of the oral mucosa is one of the only long-term or permanent side-effects. Principles, techniques, and management of oral hyperpigmentation is still not fully understood. Currently, based on observations, the only known adverse side-effect of oral hyperpigmentation is poor aesthetics. To remove oral hyperpigmentation established during chemotherapy, patients need to undergo relatively invasive treatments. For example, some of the available surgical depigmentation options include scalpel surgery, abrasion with rotary abrasives, and diode lasers [6]. Adverse side effects other than unfavorable aesthetics and less invasive treatment or preventative options need to be researched and developed.

Some of the chemotherapeutic agents known to cause hyperpigmentation may include cyclophosphamide, busulfan, bleomycin, hydroxyurea, doxorubicin, cisplatin, etoposide and fluorouracil [2,3]. However, there is lack of data and knowledge to declare chemotherapeutic drug classes associated with this sideeffect. Drug dosages causing oral hyperpigmentation should also be investigated. Moreover, prevalence of oral hyperpigmentation associated with chemotherapy remains unknown.

There are conflicting ideas as to the mechanism driving oral hyperpigmentation with chemotherapy. Proposed mechanisms include accumulation of drug or drug metabolite deposition in oral mucosa, enhanced melanin deposition with or without an increase in melanocytes, or drug-induced post inflammatory changes [2]. Understanding the mechanism tied to this side effect may facilitate better treatment options or preventative mechanisms in the future. We need to develop better strategies to help with diagnosis of oral hyperpigmentation. History or record of pigmentation prior to chemotherapy should be properly investigated. Upon initial presentation, a size measurement and photo of the lesion should be documented. Initial size and appearance information and documentation can be used to monitor the lesion over the course of time. Lesion size or color changes are important to recognize when trying to rule out the possibility of melanoma.

Chemotherapy is often the treatment of choice and first line of defense against many types of cancers. With a rise in cancer cases and chemotherapy use, dentists are likely to encounter patients with oral complications as a result of chemotherapy. Although oral hyperpigmentation is not very common, it is one of the complications that dentists may encounter. Based on current knowledge, this is a benign condition that does not significantly impact a patient's overall health or quality of life. Correct diagnosis will avoid patients concern and unnecessary biopsies. It is imperative that dentists should have the knowledge and expertise to diagnose and monitor chemotherapy-induced oral hyperpigmentation.

\section{Acknowledgement}

None.

\section{Conflict of Interest}

No conflict of interest.

\section{References}

1. Acharya S, Pai KM, Bhat S, Mamatha B, Bejadi VM, et al. (2017) Oral changes in patients undergoing chemotherapy for breast cancer. Indian J Dent Res 28(3): 261-268.

2. Sreeja C, Ramakrishnan K, Vijayalakshmi D, Devi M, Aesha I, et al. (2015) Oral pigmentation: A review. J Pharm Bioallied Sci: S403-S408. 
3. Pavey R, Kambil SM, Bhat RM (2015) Dermatological adverse reactions to cancer chemotherapy. Indian J Dermatol Venereol Leprol 81(4): $1-434$.

4. Stringer LL, Zitella L (2014) Hyperpigmentation of the tongue. J Adv Pract Oncol 5(1): 71-72.

5. Meleti M, Vescovi P, Mooi WJ, Van Der Waal I (2008) Pigmented lesions of the oral mucosa and perioral tissues: a flow-chart for the diagnosis and some recommendations for the management. Oral Surg Oral Med Oral Pathol Oral Radiol Endod 105(5): 606-616.

6. Murthy MB, KaurJ, Das R (2012) Treatment of gingival hyperpigmentation with rotary abrasive, scalpel, and laser techniques: A case series. J Indian Soc Periodontol 16(4): 614-619. 